> Pour la sixième année, dans le cadre du module d'enseignement «Physiopathologie de la signalisation » proposé par l'université Paris-sud, les étudiants du Master «Biologie Santé » de l'université Paris-Saclay se sont confrontés à l'écriture scientifique. Ils ont sélectionné une quinzaine d'articles scientifiques récents dans le domaine de la signalisation cellulaire présentant des résultats originaux, via des approches expérimentales variées, sur des thèmes allant des relations hôte-pathogène aux innovations thérapeutiques, en passant par la signalisation hépatique et le métabolisme. Après un travail préparatoire réalisé avec l'équipe pédagogique, les étudiants, organisés en binômes, ont ensuite rédigé, guidés par des chercheurs, une Nouvelle soulignant les résultats majeurs et l'originalité de l'article étudié. Ils ont beaucoup apprécié cette initiation à l'écriture d'articles scientifiques et, comme vous pourrez le lire, se sont investis dans ce travail avec enthousiasme! Trois de ces
Partenariat médecine/sciences Écoles doctorales - Masters (33)

L'actualité scientifique vue par les étudiants du Master Biologie Santé, module physiopathologie de la signalisation, Université Paris-Saclay

\begin{tabular}{l|l|l}
\hline SCHOOL & $\begin{array}{l}\text { MASTER } \\
\text { Biologie Santé } \\
\text { PARIS-SACLAY }\end{array}$ & $\begin{array}{l}\text { BIOLOGIE, MÉDECINE, } \\
\text { PHARMACIE }\end{array}$
\end{tabular}

Équipe pédagogique

Karim Benihoud (professeur, université Paris-Sud)

Sophie Dupré (maître de conférences, université Paris-Sud)

Boris Julien (maître de conférences, université Paris-Sud)

Philippe Robin (maître de conférences, université de Paris-Sud)

Hervé Le Stunff (professeur, université Paris-Sud)

karim.benihoud@u-psud.fr

Série coordonnée par Sophie Sibéril.

Nouvelles sont publiées dans ce numéro, les autres le seront dans des prochains numéros. <

\title{
NOUVELLE
}

\section{PolDIP2, une protéine clé de la régulation du fonctionnement mitochondrial et du métabolisme cellulaire}

Étienne Andjongo ${ }^{1}$, Sonia Benhamouche ${ }^{1}$, Aicha Bouraoui ${ }^{2}$ Laura Baciou ${ }^{2}$

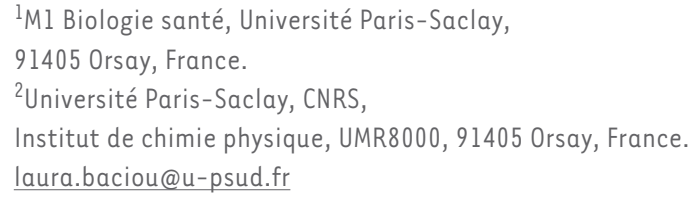




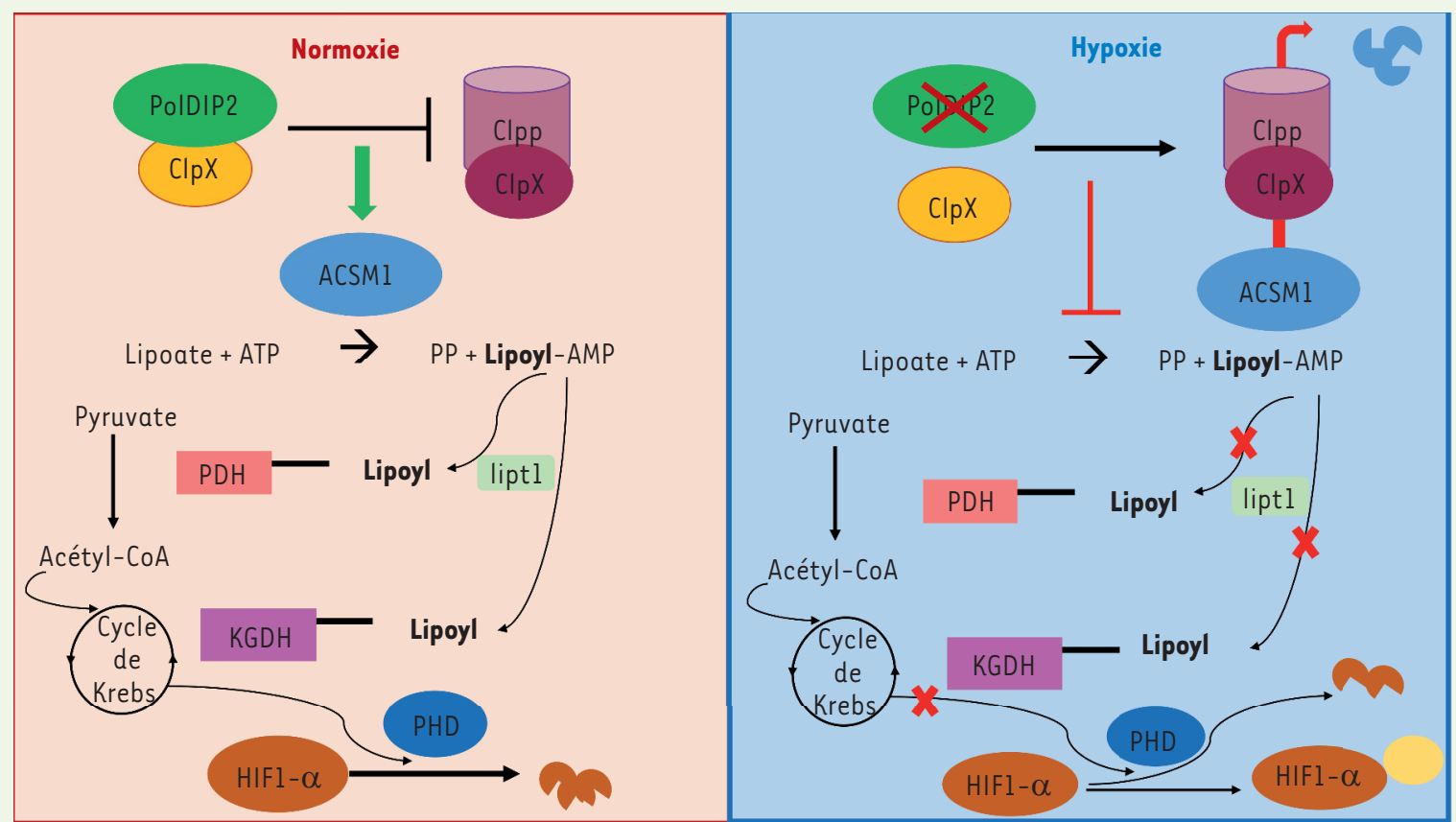

Figure 1. Rôle de PolDIP2 dans le métabolisme mitochondrial. En situation de normoxie (à gauche), PoIDIP2 séquestre Clpx, ce qui inhibe la formation du complexe Clpp-Clpx, responsable de la dégradation de la protéine mitochondriale ACSMI (Acetyl-CoA synthetase medium-chain family member 1 ). ACSMl catalyse la réaction conduisant à la formation du lipoyl-AMP à partir du lipoate, en présence d'ATP. L'enzyme mitochondriale LIPTI (lipoyltransferase 1) greffe le groupement lipoyl sur les enzymes PDH (pyruvate déshydrogénase) et $\alpha$-KGDH ( $\alpha$-cétoglutarate déshydrogénase) intervenant dans le cycle de Krebs. La respiration mitochondriale stabilise les protéines PHD (prolyl-4-hydroxylase domain-containing proteins), conduisant à la dégradation du facteur HIFl- $\alpha$.

le métabolisme cellulaire [4]. Ces auteurs ont d'abord étudié la distribution de PoIDIP2 dans différents tissus, et ont montré, dans divers types cellulaires, tels que les cellules musculaires lisses aortiques humaines, les cellules épithéliales mammaires humaines et les fibroblastes embryonnaires murins, que la protéine est localisée presque exclusivement dans les mitochondries. Ils ont ensuite comparé le métabolisme bioénergétique mitochondrial de cellules déficientes ou non en PolDIP2, et ont montré que dans les cellules exprimant faiblement PoIDIP2, le niveau d'oxygène basal est plus faible et l'acidification du milieu extracellulaire plus importante. Ils ont également découvert que ces cellules produisent moins d'ATP par phosphorylation oxydative, tout en ayant un même niveau global d'ATP [4]. Ces résultats suggèrent l'existence d'une reprogrammation cellulaire associée à une augmentation de la glycolyse anaérobie.

\section{Implication de PoIDIP2}

dans la régulation d'enzymes du cycle de Krebs

La lipoylation d'enzymes cataboliques mitochondriales, telles que les pyruvate (PDH) et $\alpha$-cétoglutarate $(\alpha-K G D H)$ déshydrogénases, est une modification post-traductionnelle de ces protéines, nécessaire à leur activité enzymatique, et consistant en une fixation covalente, par une liaison amide, d'un lipoate sur des résidus lysine spécifiques en présence d'ATP (Figure 1). Les mécanismes impliqués dans la lipoylation des protéines ont fait l'objet d'études approfondies chez la bactérie Escherichia coli [5] et, plus récemment, chez la levure Saccharomyces cerevisae [6]. Toutefois, une étude portant sur des cellules de mammifères, a montré que la lipoylation des protéines est inhibée par l'hypoxie et dans les cellules cancéreuses [7].

Paredes et al. ont entrepris de vérifier la stabilité de la protéine HIF-l $\alpha$ (hypoxia-inducible factor $1 \alpha$ ) dans les cellules déficientes ou non en PolDIP2, car HIF-l $\alpha$ est fortement exprimée en situation d'hypoxie [8]. Ils ont montré, grâce à une analyse des protéines cellulaires par western blot, qu'en l'absence de PoIDIP2, la protéine HIF-l $\alpha$ est stabilisée, ce qui induit l'expression de la pyruvate déshydrogénase kinase (PDK), un inhibiteur de la sous-unité PDHEl $\alpha$ du complexe enzymatique PDH [4].

La protéine HIF-l $\alpha$ a une demi-vie de quelques minutes. Elle est rapidement hydroxylée par un membre de la famille des prolyl-4-hydroxylases (prolyl-4-hydroxylase domain-containing proteins, PHD), puis reconnue par un complexe 
d'ubiquitine ligase $\varepsilon 3$, poly-ubiquitinylée et dégradée par le protéasome. La réaction d'hydroxylation dépend de la disponibilité en oxygène et de l' $\alpha$-cétoglutarate. Une perturbation des enzymes du cycle de Krebs inhibe les PHD et stabilise HIF-l $\alpha$. L'hypothèse des auteurs selon laquelle la stabilisation de HIF-l $\alpha$ en l'absence de PoIDIP2 serait due à l'inhibition de l'enzyme PHD2 a pu être confirmée par un dosage de l' $\alpha$-cétoglutarate, montrant que sa concentration était faible dans les cellules déficientes en PoIDIP2. De plus, l'ajout d'un dérivé de l' $\alpha$-cétoglutarate dans le milieu de culture a restauré la stabilité de HIF-l $\alpha$ [4].

Une étude génétique réalisée chez le nématode Caenorhabditis elegans avait prédit une association fonctionnelle entre les produits des gènes tag-307 et gip-2, orthologues présumés des gènes humains codant respectivement PoIDIP2 et la lipoyltransférase 1 (LIPTl) [9], une protéine mitochondriale codée par l'ADN nucléaire. Chez les mammifères, LIPTI permet le transfert du groupement lipoylAMP sur deux des trois composants enzymatiques des complexes PDH et $\alpha-K G D H$ : la dihydrolipoamide $S$-acétyltransférase (DLAT) et la dihydrolipoamide S-succinyltransférase (DLST). Paredes et al. ont analysé la lipoylation de ces deux enzymes dans les cellules déficientes en PoIDIP2, et ont trouvé une réduction significative des concentrations de lipoyl-DLAT et de lipoyl-DLST, conduisant à une inhibition des complexes enzymatiques PDH et $\alpha-K G D H$. Ils ont également constaté une augmentation, probablement par un mécanisme compensateur, mais inefficace, de la concentration de LIPTI [4].

\section{Régulation de l'enzyme ACSM1, impliquée dans la lipoylation des protéines mitochondriales, par PoIDIP2}

Il a été montré, dans des cellules de mammifère, que la lipoylation des protéines nécessite la présence de l'enzyme ACSMI (acetyl-CoA synthetase mediumchain family member 1) [10]. Paredes et al. ont observé une absence de la pro- téine ACSMI dans les cellules déficientes en PoIDIP2, associée à une diminution de la lipoylation des protéines DLAT et DLST. La lipoylation de ces deux protéines est rétablie lorsque l'expression d'ACSMI est restaurée [4]. Ces résultats indiquent que la perte d'ACSMl est la principale cause de l'absence de lipoylation dans des cellules déficientes en PoIDIP2.

Des expériences de co-immunoprécipitation ont révélé l'existence d'une interaction forte entre la protéine CLPX (caseinolytic protease subunit $X$ ) et PoIDIP2. CLPX est une ATPase chaperonne se liant à la peptidase caséinolytique CLPP pour former le complexe Clp-protéase. CLPX permet l'entrée de protéines dans le protéasome en assurant un changement de leur conformation, un processus consommant de I'ATP. Les auteurs ont montré que dans les cellules déficientes en CLPP, la diminution de l'expression de PoIDIP2 n'induit pas la dégradation de ACSMI, permettant ainsi de préserver la lipoylation des sous-unités DLAT et DLST [4]. L'interaction spécifique entre PoIDIP2 et CLPX suggère également que PoIDIP2 peut inhiber l'activité du complexe Clp-protéase en séquestrant CLPX, et empêcher par conséquent la dégradation d'ACSMI, permettant ainsi la lipoylation des complexes enzymatiques PDH et $\alpha-\mathrm{KGDH}$.

\section{Conséquence de l'hypoxie} sur le métabolisme cellulaire

Paredes et al. ont montré que l'hypoxie réduit significativement la quantité de protéines ACSMI et la lipoylation de DLAT et DLST, en même temps qu'elle induit la stabilisation de la protéine HIF-l $\alpha$. Une expression exogène de PolDIP2 en condition d'hypoxie était suffisante pour rétablir les taux d'ACSMI, de lipoyl-DLST et de lipoyl-DLAT [4].

Dans certains types de cancers agressifs, tels que le cancer du sein «triple-négatif $»^{1}$, les cellules cancéreuses présentent un phénotype hautement glycolytique.

\footnotetext{
${ }^{1}$ Cancer du sein «triple négatif » : dans cette forme du cancer du sein, les cellules tumorales n'expriment ni les récepteurs hormonaux des œstrogènes ou de la progestérone, ni le récepteur HER2.
}

Les auteurs ont émis l'hypothèse que le déficit en PoIDIP2 pourrait contribuer à ce phénotype. Ils ont démontré que, dans les cellules de cancer du sein «triple-négatif », l'expression de PoIDIP2 est faible, et la lipoylation de DLAT et DLST est complètement inhibée. Ils ont ensuite évalué l'impact de PoIDIP2 sur la fonction mitochondriale : la surexpression de PoIDIP2 dans des lignées cancéreuses a permis de stabiliser la protéine ACSMI, d'augmenter la lipoylation de la PDH et $\alpha-K G D H$, et de réduire la stabilisation d'HIF-l $\alpha$ sous condition de normoxie. Ces changements biochimiques étaient accompagnés par la lipoylation des enzymes mitochondriales, une augmentation de la respiration mitochondriale, ainsi que par une importante inhibition de la croissance cellulaire [4]. En conclusion, l'étude de Paredes et al. a permis de montrer que PoIDIP2 est un régulateur de la lipoylation de protéines mitochondriales. PoIDIP2, en séquestrant la protéine $\mathrm{Clpx}$, inhibe la formation du complexe Clpp-Clp, ce qui conduit à la stabilisation d'ACSMl, qui n'est alors plus dégradé. ACSMI catalyse la réaction conduisant à la formation du lipoyl-AMP, ce qui permet l'activation par lipoylation des enzymes PDH et $\alpha-K G D H$ intervenant dans le cycle de Krebs (Figure 1). Ce mécanisme est inhibé en condition d'hypoxie car la concentration cellulaire de PoIDIP2 diminue alors fortement. Cela permet une adaptation du métabolisme énergétique de la cellule, normale ou cancéreuse, en situation d'hypoxie. $\diamond$

PoIDIP2 regulates mitochondrial functioning and cellular metabolism

\section{LIENS D'INTÉRÊT}

Les auteurs déclarent n'avoir aucun lien d'intérêt concernant les données publiées dans cet article.

\section{RÉFÉRENCES}

1. Liu L, Rodriguez-Belmonte $\varepsilon M$, Mazloum N, et al. Identification of a novel protein, PDIP38, that interacts with the $p 50$ subunit of DNA polymerase $\delta$ and proliferating cell nuclear antigen. J Biol Chem $2003 ; 278: 10041-7$.

2. Cheng $X$, Kanki T, Fukuoh A, et al. PDIP38 associates with proteins constituting the mitochondrial DNA nucleoid. J Biochem $2005 ; 138: 673-8$. 


\section{RéFÉRENCES}

3. Kim Y, Park H, Nah J, et al. Essential role of POLDIP2 in Tau aggregation and neurotoxicity via autophagy/ proteasome inhibition. Biochem Biophys Res Commun 2015 ; 462 : 112-8

4. Paredes F, Sheldon K, Lassegue B, et al. Poldip2 is an oxygen-sensitive protein that controls PDH and $\alpha$ KGDH lipoylation and activation to support metabolic adaptation in hypoxia and cancer. Proc Natl Acad Sci USA 2018; 115 : 1789-94.

5. Morris TW, Reed KE, Cronan JE Jr. Lipoic acid metabolism in Escherichia coli: the IpIA and lipB genes define redundant pathways for ligation of lipoyl groups to apoprotein. J Bacteriol 1995 ; 177 : 1-10.

6. Hermes FA, Cronan JE. The role of the Saccharomyces cerevisiae lipoate protein ligase homologue, Lip3, in lipoic acid synthesis. Yeast $2013 ; 30: 415-27$.

7. Chen YC, Kuo CC, Chian CF, et al. Knockdown of POLDIP2 suppresses tumor growth and invasion capacity and is linked to unfavorable transformation ability and metastatic feature in non-small cell lung cancer. Exp Cell Res $2018 ; 368: 42-9$.

8. Semenza GL. Hypoxia-inducible factor l: control of oxygen homeostasis in health and disease. Pediatr Res $2001 ; 49: 614-7$.
9. Szklarczyk D, Franceschini A, Kuhn M, et al. The STRING database in 2011: functional interaction networks of proteins, globally integrated and scored. Nucleic Acids Res 2011 ; 39 : D561-8.

10. Fujiwara K, Takeuchi S, Okamura-Ikeda K, Motokawa y. Purification, characterization, and cDNA cloning of lipoate-activating enzyme from bovine liver.J Biol Chem $2001 ; 276: 28819-23$. 